\title{
Einfluss der Adaptiven Servoventilation auf das BNP bei Patienten mit Cheyne-Stokes-Atmung und leicht- bis mittelgradiger systolischer und diastolischer Herzinsuffizienz
}

Influence of Adaptive Servoventilation on B-Type Natriuretic Petide in Patients with Cheyne-Stokes Respiration and Mild to Moderate Systolic and Diastolic Heart Failure

Autoren

Institute
M. Westhoff ${ }^{1}$, M. Arzt ${ }^{2}$, P. Litterst ${ }^{1}$

Klinik für Pneumologie, Schwerpunkt Schlaf-, Beatmungs- und Intensivmedizin, Lungenklinik Hemer, Hemer

2 Zentrum für Schlafmedizin, Department für Innere Medizin II, Abteilung für Pneumologie, Universität Regensburg eingereicht 11.2.2010 akzeptiert nach Revision 15. 2. 2010

\section{Bibliografie}

DOI http://dx.doi.org/ 10.1055/s-0029-1244113

Online-Publikation: 26. 4. 2010

Pneumologie 2010; 64:

467-473 @ Georg Thieme

Verlag KG Stuttgart · New York ISSN 0934-8387

\section{Korrespondenzadresse}

\section{Dr. Michael Westhoff}

Klinik für Pneumologie, Schwerpunkt Schlaf-,

Beatmungs- und Intensivmedizin,

Lungenklinik Hemer Theo-Funccius-Str. 1 58675 Hemer

michael.westhoff@lkhemer.de

\section{Zusammenfassung \\ V}

Hintergrund: CPAP hat unterschiedliche Effekte auf eine zentrale Schlafapnoe mit Cheyne-Stokes-Atmung (CSA-CSR), während die Adaptive Servoventilation (ASV) effektiver in der Normalisierung der Atmung bei Herzinsuffizienzpatienten ist. Ziel der Untersuchung war es nachzuweisen, dass eine AHI-Normalisierung durch ASV erhöhte BNP-Werte auch von Patienten mit milder systolischer und diastolischer Herzinsuffizienz reduziert.

Methode: Von April 2004 bis Oktober 2006 wurden Patienten mit CSA-CSR - ohne und mit begleitender obstruktiver Schlafapnoe (OSA) -, klinischen Zeichen der Herzinsuffizienz - unabhängig von der EF - und erhöhten BNP-Werten $(>100 \mathrm{pg} / \mathrm{ml})$ mit ASV therapiert, sofern CPAP nicht zu einer AHI-Reduktion < 15/h geführt hatte. Nach 6 Wochen erfolgten eine Polysomnografie und BNP-Bestimmung.

Resultate: 15 männliche Patienten (AHI 48,3 $\pm 14,6 / \mathrm{h}$ ) erfüllten die Einschlusskriterien; 7 hatten eine CSA-CSR + OSA, 8 eine CSA-CSR. Nach 6wöchiger ASV-Therapie sank das BNP von 415 $\pm 196 \mathrm{pg} / \mathrm{ml}$ auf $264 \pm 146 \mathrm{pg} / \mathrm{ml}(\mathrm{p}=0,0009)$. Die BNP-Reduktion war nur signifikant in der CSACSR + OSA Subgruppe $(\mathrm{p}=0,0002)$.

Schlussfolgerung: ASV kann den AHI von Patienten mit milder systolischer und diastolischer Herzinsuffizienz und CSA-CSR \pm OSA normalisieren. Dies führt zu einer signifikanten BNP-Reduktion und legt nahe, dass auch in dieser Herzinsuffizienzgruppe eine Beseitigung der zentralen Schlafapnoe eine Verbesserung der Herzfunktion bewirkt.

\section{Abstract \\ V}

Background: CPAP therapy has a variable effect on central sleep apnea with Cheyne-Stokes respiration (CSA-CSR). Adaptive servoventilation (ASV) is more effective in normalising breathing in patients with heart failure. We hypothesised that, by normalising AHI, ASV reduces elevated BNP levels in patients with mild systolic and diastolic heart failure.

Methods: From April 2004 to October 2006, patients with CSA-CSR with and without concomitant obstructive sleep apnea (OSA), clinical evidence of heart failure, regardless $\mathrm{EF}$, and elevated BNP levels ( $>100 \mathrm{pg} / \mathrm{mL}$ ) were selected for treatment with ASV, unless CPAP therapy had reduced AHI to $<15$ per hour of sleep. Follow-up polysomnographies and BNP analyses were performed after 6 weeks.

Results: 15-male patients (AHI $48.3 \pm 14.6 / \mathrm{h}$ ) fulfilled all inclusion criteria; 7 patients had CSACSR + OSA, 8 had CSA-CSR. After 6 weeks of ASV, BNP decreased from $415 \pm 196 \mathrm{pg} / \mathrm{mL}$ to 264 $\pm 146 \mathrm{pg} / \mathrm{mL}(\mathrm{p}=0.0009)$. There was only a significant BNP reduction in the CSA-CSR+OSA subgroup $(\mathrm{p}=0.0002)$.

Conclusion: ASV can normalise AHI in patients with mild systolic and diastolic heart failure and CSA-CSR \pm OSA, thus leading to a significant reduction of BNP levels. These findings suggest that effective suppression of sleep apnoea in such heart failure patients improves cardiac function. 


\section{Einleitung \\ $\nabla$}

Epidemiologische Daten zeigen eine hohe Prävalenz der Schlafapnoe bei Patienten mit Herzinsuffizienz. In Abhängigkeit vom NYHA-Stadium und den diagnostischen Methoden variiert sie zwischen 53\% [1] and 76\% [2]. Das Auftreten einer Cheyne-Stokes-Atmung mit zentraler Schlafapnoe (CSA-CSR), die einen Marker für den Schweregrad und die Prognose der Herzinsuffizienz darstellt, wird in $15 \%$ [3] bis $40 \%$ beobachtet [4]. Neben den reinen Formen mit OSA auf der einen und CSA-CSR auf der anderen Seite gibt es eine Koexistenz von obstruktiver Schlafapnoe (OSA) und CSA-CSR bei Herzinsuffizienz [5,6]. Das OSA verschlechtert die Prognose bei Herzinsuffizienz und erhöht die Empfänglichkeit für das Auftreten einer CSA-CSR. Eine CPAP-Therapie führt bei Patienten mit Herzinsuffizienz und OSA zu einer Verbesserung der kardialen Funktion und Prognose [7-11], hat aber nur einen variablen Einfluss auf CSA-CSR. Der „Canadian Continuous Positive Airway Pressure for Patients with Central Sleep Apnea and Heart Failure Trial“" (CANPAP trial) hat gezeigt, dass eine längerfristige CPAP-Behandlung nur in etwa 50\% eine CSA-CSR bei Herzinsuffizienz beseitigen kann. Bei nur inkompletter Beseitigung der schlafbezogenen Atemstörung verbessert CPAP die linksventrikuläre Funktion (LVEF) nur mäßig und zeigt keinen signifikanten Effekt auf die Mortalität [12]. Allerdings zeigt eine Subgruppenanalyse, dass bei früher Normalisierung der CSA-CSR durch CPAP eine signifikante Verbesserung der LVEF und des transplantationsfreien Überlebens auftritt [13].

Diese [13] sowie frühere Daten [14] legen nahe, dass eine effektive Behandlung der Schlafapnoe eine Schlüsselrolle in der Verbesserung des kardiovaskulären Outcomes darstellt.

Die Adaptive Servoventilation (ASV) ist effektiver in der Normalisierung der Atmung bei Patienten mit Herzinsuffizienz und CSACSR, ob mit oder ohne begleitende OSA, als eine reine CPAP Therapie [15-19]. Sie gewährleistet eine Durchgängkeit der oberen Atemwege durch einen suffizienten exspiratorischen Atemwegsdruck (EPAP), um obstruktive ventilatorische Ereignisse zu behandeln. Darüber hinaus leistet sie eine inspiratorische Druckunterstützung, die invers zur Atmung des Patienten ist. Dies reduziert die Gesamtventilation, erhöht dadurch den arteriellen $\mathrm{pCO}_{2}$ und vermeidet $\mathrm{pCO}_{2}$-Absenkungen unter die Apnoeschwelle [15]. Auf diese Weise stabilisiert sie die Ventilation und bietet ein pathophysiologisch basiertes Konzept zur effektiven Behandlung der CSA-CSR.

Wir haben deshalb untersucht, ob 1. ASV die Atmung von Herzinsuffizienzpatienten mit erhöhtem BNP und CSA-CSR - unabhängig von der EF - normalisiert und 2. die Normalisierung der Atmung sich günstig auf die neurohumorale Aktivierung auswirkt, indem es ein erhöhtes BNP, das einen sensitiven und prognostischen Marker sowohl einer diastolischen als auch systolischen Herzinsuffizienz darstellt [20-22], signifikant reduziert. Da die obstruktive Schlafapnoe selbst einen negativen Einfluss auf die Herzinsuffizienz hat, wurde zusätzlich eine Subgruppenanalyse der CSA-CSR-Patienten mit und ohne begleitende obstruktive Ereignisse (CSA-CSR + OSA und CSA-CSR) durchgeführt.

\section{Material and Methoden}

$\nabla$

\section{Studiendesign}

Im Zeitraum vom 1.4.2004 bis 31.10.2006 wurden Patienten, die in der Nativpolysomnografie eine CSA-CSR mit oder ohne OSA zeigten, klinische Zeichen einer Herzinsuffizienz boten und ein erhöhtes BNP (Biosite ${ }^{\circledR}$ ) aufwiesen, für eine Behandlung mit ASV vorgesehen, wenn eine CPAP-Therapie (Druckbereich 510 mbar) über 2 Nächte nicht zu einer Normalisierung des Atemmusters geführt hatte. Die Patienten gaben ihr schriftliches Einverständnis. Das BNP wurde nach 6-wöchiger ASV-Therapie kontrolliert. In die endgültige Auswertung wurden nur Patienten mit einer Mindestnutzung der ASV-Therapie von 4 Stunden/Nacht eingeschlossen.

\section{Ein- und Ausschlusskriterien}

Die Einschlusskriterien für die klinische Evaluation waren: (1) stabile Herzinsuffizienz NYHA I-III aufgrund ischämischer, idiopathischer dilatativer oder hypertensiver Kardiomyopathie (die Diagnose der Herzinsuffizienz erfolgte gemäß der aktuellen Leitlinie der Europäischen Gesellschaft für Kardiologie [23]), (2) BNP > $100 \mathrm{pg} / \mathrm{ml}$, (3) CSA-CSR (zentraler Apnoe-Index (CAI) > 5/h; zentraler Apnoe-Hypopnoe-Index (CAHI) > 15/h) unter CPAP; (4) Apnoe-Hypopnoe-Index (AHI) < 15/h unter ASV; (5) Nutzung der ASV > 4 h/Nacht; (6) unveränderte kardiale Medikation seit 8 Wochen vor dem Einschluss in die Untersuchung. Ausschlusskriterien waren: (1) klinische Zeichen einer dekompensierten Herzinsuffizienz, (2) Krankenhausaufenthalt wegen Herzinsuffizienz innerhalb 2 Monaten vor Aufnahme in das Schlaflabor, (3) vorausgegangene CPAP- oder Bilevel-Therapie, (4) Nutzung der ASV $<4$ h/Nacht.

\section{Protokoll}

Alle Patienten wurden im Schlaflabor einer Nativ-Polysomnografie (PSG) unterzogen. In der zweiten Nacht erfolgte eine CPAP-Titration mit einem auto-adaptiven System (Somnosmart ${ }^{\circledR}$ II, Fa. Weinmann), das auf einer Oszilloresistometrie mit Analyse von Druck, Flow und Schnarchereignissen basiert. In einer weiteren Therapienacht wurde unter PSG-Aufzeichnung und unter Zugrundelegung der Ergebnisse der ersten Therapienacht eine Einstellung mit einem festen CPAP-Druck gewählt, der obstruktive Ereignisse effektiv beseitigte.

ASV (Auto-CS ${ }^{\circledR}$ - Fa. Resmed, Australien) wurde unter polysomnografischer Kontrolle eingesetzt. Als exspiratorischer positiver Atemwegsdruck (EPAP) wurde der Druck gewählt, der im Rahmen der CPAP-Therapie obstruktive Apnoen und Hypopnoen sowie bei gemischtförmigen Apnoen die obstruktiven Anteile effektiv unterdrückte. Die inspiratorische Druckunterstützung wurde auf 3-10 $\mathrm{cm} \mathrm{H}_{2} \mathrm{O}$ mit einer Atemfrequenz von 15/min eingestellt.

\section{Polysomnografie}

Die Polysomnografie wurde mit dem Alice $3.5^{\circledR}$ System (Respironics, Murrysville, PA) durchgeführt. Folgende Parameter wurden aufgezeichnet: EEG C4A1 und C3A2, submentale und prätibiale Elektromyografie, Elektrooculografie, EKG, Atembewegungen (thorakale und abdominelle Induktionsplethysmografie), Atemfluss mit Druckflow-Monitor (Heinen und Löwenstein, Bad Ems, Germany) und mit einem oralen/nasalen Thermistor (Respironics, Murrysville, PA), Schnarchereignisse (Alice ${ }^{\circledR}$ laryngeales Mikrofon und Backelektret Mikrofon Typ ME 2/V 413, Peiker Inc.), Sauerstoffsättigung mittels Fingerpulsoxymetrie (Fa. Novametrix $\left.{ }^{\circledR}\right)$. Der Luftfluss während der PSG unter CPAP und ASV wurde mit einem Druckflowmonitor erfasst.

Die PSGs wurden manuell ausgewertet (M.W. und P.L). Schlafstudien- und Arousal-Analyse erfolgten nach Rechtschaffen und Kales [24] und den Empfehlungen der American Sleep Disorders Association [25]. 


\begin{tabular}{|c|c|c|c|c|c|}
\hline Variablen & $\begin{array}{l}\text { Alle Patienten } \\
n=15\end{array}$ & $\begin{array}{l}\text { CSA-CSR + OSA } \\
n=7\end{array}$ & $\begin{array}{l}\text { CSA-CSR } \\
n=8\end{array}$ & p-Wert & $\begin{array}{l}\text { Tab. } 1 \text { Anthropometrische } \\
\text { und echokardiografische Da- }\end{array}$ \\
\hline Alter (Jahre) & $68,9 \pm 7,1$ & $71,1 \pm 5,6$ & $66,9 \pm 8,0$ & $0,26=n . s$. & $\begin{array}{l}\text { ten, ESS und } \mathrm{pCO}_{2} \text { im gesamten } \\
\text { Kollektiv und unterteilt nach }\end{array}$ \\
\hline $\operatorname{BMI}\left(\mathrm{kg} / \mathrm{m}^{2}\right)$ & $31,3 \pm 5,0$ & $32,9 \pm 6,3$ & $29,9 \pm 3,1$ & $0,26=n . s$. & Subgruppen (CSA-CSR + OSA \\
\hline ESS & $9,7 \pm 5,0$ & $12,0 \pm 3,6$ & $7,4 \pm 4,8$ & $0,06=n . s$. & und CSA-CSR) (n. s. = nicht sig- \\
\hline $\operatorname{LA}(\mathrm{cm})$ & $4,9 \pm 0,6$ & $4,6 \pm 0,4$ & $5,2 \pm 0,6$ & 0,04 & nifikant). \\
\hline $\operatorname{LVEF}(\%)$ & $47,8 \pm 10,3$ & $45,9 \pm 10,8$ & $49,5 \pm 10,3$ & $0,54=n . s$ & \\
\hline $\mathrm{pCO}_{2}(\mathrm{~mm} \mathrm{Hg})$ & $35,6 \pm 3,1$ & $36,5 \pm 2,8$ & $34,6 \pm 3,3$ & $0,28=n . s$. & \\
\hline
\end{tabular}

Eine zentrale Apnoe wurde definiert als ein Fehlen von Atemfluss für $\geq 10 \mathrm{~s}$, assoziiert mit einem Fehlen thorakaler und abdomineller Exkursionen; eine zentrale Hypopnoe als eine Reduktion des Atemflusses um mindestens $50 \%$ gegenüber dem Ausgangsflow für $\geq 10$ s oder eine Amplitudenreduktion von $<50 \%$ zusammen mit entweder einer Sauerstoffdesaturation von $>3 \%$ oder einem Arousal, beides ohne Hinweis auf eine Flusslimitation. Eine obstruktive Apnoe wurde definiert als ein Fehlen von Atemfluss für $\geq 10$ s bei erhaltenen Atemanstrengungen und eine obstruktive Hypopnoe als eine Atemflussreduktion um mindestens $50 \%$ im Vergleich zum Ausgangsflow für $\geq 10$ s oder ein Amplitudenreduktion von $<50 \%$ assoziiert entweder mit einer Desaturation von $>3 \%$ oder einem Arousal. Gemischte Apnoen wurden definiert als Apnoen von $\geq 10$ s Dauer, die sowohl zentrale als auch obstruktive Anteile aufwiesen. Schnarchen, das mittels einer online Audio-Video-Aufzeichnung nachgewiesen wurde, stellte ein zusätzliches Kriterium für eine begleitende obstruktive Komponente bei gemischten Apnoen und Hypopnoen dar. Der Apnoe/ Hypopnoe-Index (AHI) wurde definiert als die gesamte Zahl von Apnoen und Hypopnoen im Verhältnis zur gesamten Schlafzeit. Die Polysomnografie wurde in einem von der DGSM akkreditierten Schlaflabor durchgeführt.

\section{Statistische Analyse}

Die Werte wurden als Mittelwerte \pm Standardabweichung berechnet. Für das gesamte Untersuchungskollektiv sowie für die Untergruppen (CSA-CSR mit und ohne OSA) wurden die präund posttherapeutischen Werte von BNP, AHI, Arousal-Index, Schlafstadien, ESS auf Normalverteilung geprüft und mittels paarigem Student-t-Test verglichen. Signfikanz wurde bei einem $\mathrm{p}<0,05$ festgesetzt.

\section{Ergebnisse \\ $\nabla$}

\section{Patienten- und Schlafdaten}

Im Beobachtungszeitraum wurde bei 1348 Patienten die Diagnose einer behandlungspflichtigen schlafbezogenen Atemstörung gestellt. Davon hatten 31 Patienten (2,3\%) auch nach CPAP-Therapie noch eine zentrale Atemstörung vom Cheyne-Stokes Typ und ein erhöhtes BNP. Fünf Patienten erfüllten wegen kardialer Dekompensation nicht die Einschlusskriterien, 2 weitere Patienten mussten wegen Intoleranz der CPAP-Therapie bzw. Ablehnung der ASV ausgeschlossen werden. 24 Patienten unterzogen sich der ASV-Therapie. 3 Patienten tolerierten die ASV-Therapie nicht, 1 Patient hatte nach 6 Wochen einen AHI >15/h, 2 Patienten nutzten das Gerät weniger als $4 \mathrm{~h} / \mathrm{Nach}$ t und 3 lehnten eine weitere Teilnahme an der Untersuchung ab, so dass 15 Patienten in die Auswertung eingingen.

Von diesen verbleibenden männlichen Patienten hatten 8 eine reine CSA-CSR und 7 eine CSA-CSR mit zusätzlichen obstruktiven bzw. gemischtförmigen Apnoen in der Nativ-PSG ( $\bullet$ Tab. 1). Zwi-
Tab. 2 Erkrankungen aller Patienten und unterteilt nach Subgruppen (CSA-CSR + OSA und CSA-CSR).

\begin{tabular}{|lcll|}
\hline Variablen & Alle Patienten & CSA-CSR+OSA & CSA-CSR \\
& $\mathbf{n}(\%)$ & $\mathbf{n}(\%)$ & $\mathbf{n}(\%)$ \\
\hline Vorhofflimmern & $9(60)$ & $3(43)$ & $6(67)$ \\
\hline Hypertonus & $13(87)$ & $7(100)$ & $6(75)$ \\
\hline Diabetes & $7(47)$ & $4(57)$ & $3(38)$ \\
\hline KHK & $12(80)$ & $5(71)$ & $7(88)$ \\
\hline
\end{tabular}

Tab. 3 Kardiale Medikation aller Patienten und unterteilt nach Subgruppen (CSA-CSR + OSA und CSA-CSR) - (n. s. = nicht signifikant).

$\begin{array}{llll}\text { Variablen } & \text { Alle Patienten } & \text { CSA-CSR + OSA } & \text { CSA-CSR } \\ & \mathbf{n}(\%) & \mathbf{n}(\%) & \mathbf{n}(\%) \\ \beta \text {-Blocker } & 14(93) & 6(86) & 8(100) \\ \text { ACE } & 15(100) & 7(100) & 8(100) \\ \text { Diuretika } & 14(93) & 7(100) & 7(88) \\ \text { Digitalis } & 4(27) & 2(28) & 2(25) \\ \text { Ca-Antagonist } & 5(33) & 3(43) & 2(25)\end{array}$

schen den Subgruppen ergaben sich keine signifikanten Unterschiede im Alter, BMI, ESS und in der LVEF. Der linke Vorhof (LA) war in der CSA-CSR Gruppe größer $(5,2 \pm 0,6)$ als in der CSA-CSR + OSA Gruppe $(4,6 \pm 0,4)(\mathrm{p}=0,04)(-$ Tab. 2$)$.

Die Therapie der Herzinsuffizienz erfolgte leitliniengerecht mit B-Blockern, ACE-Hemmern und Diuretika im Gesamtkollektiv und den Subgruppen ( $\bullet$ Tab. 3 ).

AHI, Arousal-Index und Schlafstadien unterschieden sich in den Subgruppen nicht signifikant, während die Desaturationen in der Subgruppe mit begleitenden obstruktiven Ereignissen ausgeprägter waren $(\mathrm{p}=0,04)(\bullet$ Tab. 4).

Im Rahmen der CPAP-Therapie wurden bei allen Patienten CPAPDrucke bis 10 mbar appliziert, ohne dass bei den in die Studie eingeschlossenen Patienten die zentralen Ereignisse beseitigt werden konnten. Unter einem mittleren CPAP von 5,6 $\pm 1,0 \mathrm{~cm}$ $\mathrm{H}_{2} \mathrm{O}$ waren die obstruktiven Apnoen und obstruktiven Anteile der gemischtförmigen Apnoen in der CSA-CSR + OSA Gruppe effektiv unterdrückt und es persistierte eine typische Cheyne-Stokes-Atmung. Der AHI sank darunter auf 37,0 $\pm 16,1 / \mathrm{h}$ und entsprach damit dem AHI der CSA-CSR-Gruppe unter CPAP $(36,7$ $\pm 15,5 \mathrm{n} / \mathrm{h})$.

\section{Effekte von ASV auf AHI und BNP}

Die ASV-Therapie mit einem EPAP von 5,6 $\pm 1,0 \mathrm{~cm} \mathrm{H}_{2} \mathrm{O}$, einer Druckunterstützung von $3-10 \mathrm{~cm} \mathrm{H}_{2} \mathrm{O}$ und einer back-up Atemfrequenz von 15/min normalisierte den AHI in der ersten Nacht auf $4,4 \pm 5,5 / h$.

Nach 6-wöchiger ASV-Therapie und einer durchschnittlichen nächtlichen Nutzung von $5,5 \pm 1,0 \mathrm{~h}$ zeigten sich der AHI $(2,8$ $\pm 3,8 / \mathrm{h}-\mathrm{p}<0,001)$ und der Arousal-Index $(16,5 \pm 9,5 / \mathrm{h}-\mathrm{p}<0,01)$ normalisiert $(\bullet$ Abb. 1). 


\begin{tabular}{|c|c|c|c|c|}
\hline Variablen & $\begin{array}{l}\text { Alle Patienten } \\
n=15\end{array}$ & $\begin{array}{l}\text { CSA-CSR + OSA } \\
n=7\end{array}$ & $\begin{array}{l}\text { CSA-CSR } \\
n=8\end{array}$ & p-Wert \\
\hline $\mathrm{AHI}(\mathrm{n} / \mathrm{h})$ & $48,3 \pm 14,6$ & $55,6 \pm 9,0$ & $42,0 \pm 16,2$ & $0,07=$ n.s. \\
\hline $\mathrm{CAI}(\mathrm{n} / \mathrm{h})$ & $14,8 \pm 12,8$ & $11,5 \pm 15,4$ & $17,3 \pm 10,8$ & $0,42=n . s$. \\
\hline $\mathrm{OAI}+\mathrm{MAI}(\mathrm{n} / \mathrm{h})$ & $11,1 \pm 12,6$ & $22,0 \pm 10,4$ & $1,6 \pm 1,4$ & 0,0006 \\
\hline $\mathrm{CHI}$ & $16,5 \pm 13,3$ & $10,0 \pm 10,2$ & $22,2 \pm 13,7$ & $0,21=$ n.s. \\
\hline $\mathrm{OHI}$ & $6,8 \pm 11,6$ & $13,7 \pm 14,5$ & $0,8 \pm 1,6$ & 0,007 \\
\hline Arousals (n/h) & $45,5 \pm 20,9$ & $44,9 \pm 14,8$ & $46,1 \pm 26,1$ & $0,92=$ n.s. \\
\hline NREM $1 / 2(\%)$ & $80,7 \pm 11,1$ & $82,2 \pm 9,5$ & $79,3 \pm 13,0$ & $0,64=$ n.s. \\
\hline NREM 3 / 4 (\%) & $8,7 \pm 10,8$ & $3,9 \pm 6,0$ & $12,9 \pm 12,6$ & $0,11=$ n.s. \\
\hline REM (\%) & $9,3 \pm 6,1$ & $9,7 \pm 7,1$ & $9,0 \pm 5,5$ & $0,83=$ n.s. \\
\hline Nadir $\mathrm{SaO}_{2}(\%)$ & $76,3 \pm 10,0$ & $70,9 \pm 8,4$ & $81,0 \pm 9,2$ & 0,04 \\
\hline
\end{tabular}

Tab. 4 Schlafdaten aller Patienten und unterteilt nach Subgruppen (CSA-CSR + OSA und CSA-CSR) - (n. s. = nicht signifikant).

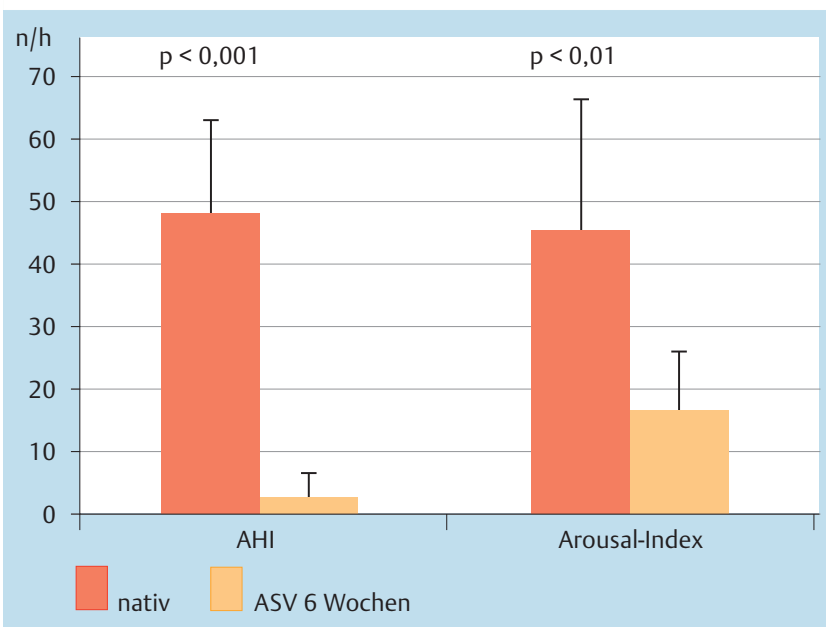

Abb. 1 BNP bei allen Patienten $(n=15)$ vor und nach 6 Wochen ASV-Therapie.

Die Reduktion der Tagesschläfrigkeit (ESS-score: von 9,7 $\pm 5,0$ auf $7,9 \pm 5,0, \mathrm{p}=0,40$ ) und die Änderung der Schlafstadienverteilung (NREM $1 / 2: 80,7 \pm 11,2 \%$ auf $69,3 \pm 24,9 \%$, NREM $3 / 4: 8,7 \pm 10,8 \%$ auf $14,2 \pm 20,7 \%$ und REM-Schlaf: $9,3 \pm 6,1 \%$ auf $13,8 \pm 9,0 \%$ ) waren statistisch nicht signifikant.

Das BNP zeigte einen signifikanten Rückgang von $415 \pm$ $196 \mathrm{pg} / \mathrm{ml}$ auf $264 \pm 146 \mathrm{pg} / \mathrm{ml}(\mathrm{p}=0,0009)$ im Gesamtkollektiv (๑ Abb. 2).

Obwohl der AHI und Arousal-Index in beiden Subgruppen normalisiert war, war die BNP-Reduktion in der Subgruppe der Patienten mit begleitenden obstruktiven bzw. gemischtförmigen Ereignissen ausgeprägter; so zeigte sich ein BNP-Rückgang von $518,3 \pm 208,9$ auf $269,6 \pm 156,1 \mathrm{pg} / \mathrm{ml}(\mathrm{p}=0,0002)$. Die prätherapeutischen BNP-Spiegel der CSA-CSR-OSA-Gruppe lagen im Vergleich zu der CSA-CSR-Gruppe durchschnittlich höher, posttherapeutisch bestand kein Unterschied. In der CSR-CSA-Subgruppe ohne OSA wiesen 7 von 8 Patienten eine BNP-Reduktion auf, während 1 Patient trotz regelmäßiger Nutzung der ASV-Therapie, Normalisierung des AHI und klinischer Verbesserung einen Anstieg des BNP aufwies. Die BNP-Reduktion unter Einschluss aller Patienten der Subgruppe (324,6 $\pm 139,7$ auf 259,4 $\pm 147,6$ pg/ $\mathrm{ml})$ zeigte zwar einen Trend, war jedoch nicht $(\mathrm{p}=0,15)$ signifikant. Bei keinem der Patienten kam es unter der Therapie zu einer Änderung des Herzrhythmus.

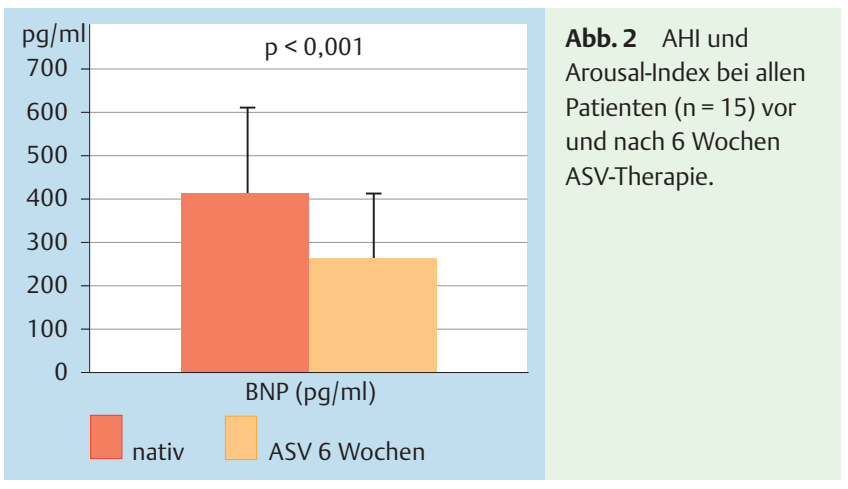

\section{Diskussion}

Die Daten zeigen, dass die ASV-Therapie bei Patienten mit erhöhtem BNP-Spiegel infolge einer Herzinsuffizienz und begleitender CPAP-resistenter zentraler Schlafapnoe vom Cheyne-Stokes-Typ den AHI normalisiert. Gleichzeitig kommt es zu einer signfikanten Reduktion erhöhter BNP-Spiegel nach einer Therapiedauer von 6 Wochen. Dies betrifft Patienten mit systolischer und diastolischer Herzinsuffizienz - unabhängig von der EF -, die die ASV-Therapie mehr als 4 Stunden in der Nacht nutzen. Die Normalisierung der Atmung bewirkt einen günstigen Effekt auf die neurohumorale Aktivierung, insbesondere auch bei Patienten mit einer Cheyne-Stokes-Atmung und einer EF $>40 \%$. Weiterhin zeigen die Daten, dass Patienten mit Herzinsuffizienz neben reinen zentralen Apnoen einen hohen Anteil von Hypopnoen und zumindest in einer Subgruppe auch einen relevanten Anteil obstruktiver bzw. gemischt zentral-obstruktiver Apnoen aufweisen.

Neue Konzepte in der Interpretation der Herzinsuffizienz und der Schlafapnoe gehen davon aus, dass 1. im Falle einer Koexistenz der Effekt der Schlafapnoe sowie der Herzinsuffizienz auf das sympathische Nervensystem additiv ist und 2. die Behandlung der Schlafapnoe bei diesen Patienten eine Abschwächung der Aktivierung des sympathischen Nervensystems herbeiführt [26]. Mehrere Marker der neurohumoralen Aktivierung finden sich bei Patienten mit Herzinsuffizienz und Schlafapnoe hochreguliert. Solin et al. [27] zeigten, dass Herzinsuffizienzpatienten eine höhere Norepinephrinauscheidung im Urin als Patienten mit Schlafapnoe aufweisen. Allerdings hatte eine OSA keinen weiteren Einfuss auf die Norepinephrinausscheidung bei Herzinsuffizienz und eine Subgruppe mit CSA-CSR wurde nicht untersucht. Plasmaendothelinspiegel [28] und BNP [29,30] wurden bei Herzinsuffizienz und CSA-CSR erhöht gefunden. Das BNP zeigt eine unabhängige und signifikante Korrelation mit dem 
AHI, dem Desaturationsindex und der Schwere der kardialen Erkrankung [31,32]. Es wird als der zweidimensionalen Echokardiografie überlegen in der Identifikation einer Herzinsuffizienz beschrieben [31] und stellt einen sensitiven Marker nicht nur für die systolische, sondern auch für die diastolische Herzinsuffizienz dar [20 - 22]. Aus diesen Gründen wurde in der eigenen Untersuchung das erhöhte BNP als Marker für die Schwere der Herzinsuffizienz herangezogen. Dies wird unterstützt durch neuere Ergebnisse, die zeigen, dass Veränderungen der BNP-Werte signifkant mit der klinischen Verbesserung unter einer Herzinsuffizienztherapie korrelieren [32].

Zu Studienbeginn lagen keine vergleichbaren Literaturdaten über den Einfluss der ASV-Therapie auf das BNP bei Patienten mit milder systolischer und diastolischer Herzinsuffizienz vor. Die meisten therapeutischen Interventionen bei Herzinsuffizienz und CSA-CSR wurden bei Patienten mit mäßiger und schwerer systolischer Herzinsuffizienz, festgelegt durch eine LVEF $<40 \%$, durchgeführt [12,15-19]. Pepperell et al [16] berichteten über einen signifikanten BNP-Rückgang von 56 pg/ml und ein Absinken der Urinausscheidung von Metadrenalin unter der ASV-Therapie im Vergleich zu einer subtherapeutischen ASV-Therapie bei Patienten mit Herzinsuffizienz mit CSA-CSR und einer EF $<40 \%$. Oldenburg et al. [33] beobachteten einen signifikanten Rückgang von NT-pro-BNP durch ASV. Dies war assoziiert mit einem Anstieg der LVEF, der Belastbarkeit und der peak- $\mathrm{VO}_{2}$ während einer Spiroergometrie. Aber auch hier handelte es sich durchweg um Patienten mit einer LVEF $<40 \%$.

Allerdings ist eine CSA-CSR nicht nur auf Patienten mit einer $\mathrm{EF}<40 \%$ beschränkt, sondern wird auch bei einer Herzinsuffizienz mit einer LVEF $>40 \%$ oder sogar bei reiner diastolischer Herzinsuffizienz beobachtet. Diese Patientengruppe ist aber bislang nicht entsprechend untersucht, obwohl sie einen vergleichbaren Schweregrad der Atemstörung und auch relevante BNP-Erhöhungen aufweisen kann. Aus diesem Grunde erfolgte die Selektion der Patienten für die dargestellte Untersuchung bewusst allein durch das Auftreten einer CSA-CSR und einer damit gekoppelten BNP-Erhöhung. Darüber hinaus spiegelt das gewählte Kollektiv die reale Situation in einem Schlaflabor wider.

Klinische Daten zeigen eine erhöhte Mortalität bei Herzinsuffizienz und OSA mit einem AHI > 15/h [34] und eine signifikante Reduktion der Mortalität von Herzinsuffizienzpatienten durch eine Therapie der OSA mit CPAP [7,8]. Eskafi et al. [35] berichteten, dass bei Herzinsuffizienzpatienten die Behandlung einer Schlafapnoe mit einer Protrusionschiene sogar zu einer Reduktion des BNP um 47,7 pg/ml führt, obwohl der AHI nicht komplett normalisiert wurde. Deshalb ist das Ausmaß einer BNP-Erhöhung bei Patienten mit Herzinsuffizienz und CSA-CSR auch durch eine koexistente OSA erklärbar. Epidemiologische Daten über die Prävalenz der Schlafapnoe bei Herzinsuffizienz beschränken sich weitgehend auf eine Unterscheidung von CSA-CSR und OSA [1$3,36,37$ ]. Oldenburg et al. [2] schließen eine relevante Überlappung von CSA-CSR und OSA aus, wobei die Ergebnisse auf polygrafischen Untersuchungen basieren, die nach Apelt et al. [42] in der Diagnostik schlafbezogener Atemstörung bei Patienten mit Herzinsuffizienz der Polysomnografie an Genauigkeit unterlegen ist. Darüber hinaus muss eine relevante obstruktive Komponente nicht zwangsläufig einen Einfluss auf die dominante Form der Atemstörung haben. Obwohl die Koexistenz von OSA und CSA bei Herzinsuffizienzpatienten beschrieben ist, ist diese Subgruppe der Patienten mit CSA-CSR nicht genauer untersucht.

Unsere Daten legen nahe, dass eine BNP-Reduktion bei CSA-CSR im Zusammenhang mit der Koexistenz begleitender obstruktiver
Ereignisse, die sich bei den Patienten der Studie vorwiegend in Form gemischtförmiger Apnoen manifestierte, diskutiert werden sollte. So bestand nur für die Gesamtgruppe und die Subgruppe mit CSA-CSR + OSA eine signifikante BNP-Reduktion, während sich für die Subgruppe mit reiner CSA-CSR nur ein deutlicher Trend in der BNP-Reduktion zeigte. Da die posttherapeutischen BNP-Werte für beide Subgruppen nicht signifikant unterschiedlich waren, ist die Absenkung durch die primär höheren prätherapeutischen BNP-Spiegel in der OSA-Subgruppe zu erklären. Dies wiederum kann als Hinweis gewertet werden, dass das Auftreten von Obstruktionen der oberen Atemwege bei CSA-CSR zu einer zusätzlichen neurohumoralen Aktivierung mit vermehrter BNP-Ausschüttung führt.

Diese Beobachtungen stimmen mit früheren Untersuchungsergebnissen überein, nach denen eine Therapie mit positivem Atemwegsdruck die kardiale Funktion von Herzinsuffizienzpatienten deutlicher bei prädominant obstruktiven Ereignissen zu verbessern scheint $[9,11]$ als bei Patienten mit prädominanter CSA [12,38].

Während Oldenburg et al. [39] nur eine geringe Nacht-zu-NachtVariabilität von schlafbezogenen Atemstörungen bei Einsatz der Polygrafie sahen, berichten andere Autoren [40-42] relevante Nacht-zu-Nacht-Veränderungen im Schlafapnoe-Typ. Tkacova et al. [5] beschrieben einen „overnight shift“ des Schlafapnoetyps bei Patienten mit Herzinsuffizienz. Deshalb wurde in der eigenen Untersuchung vor Initiierung der ASV-Therapie mindestens eine Diagnostiknacht mit einer Nativ-Polysomnografie einschließlich einer Audio-Video-Dokumentation zur Interpretation der zugrundeliegenden Atemstörungen eingesetzt. Dieses Vorgehen findet seine Bestätigung durch die Ergebnisse einer aktuellen Untersuchung von Apelt et al. [42] zur Genauigkeit von Polygrafie und Polysomnografie bei der Diagnostik von Atemstörungen bei Patienten mit Herzinsuffizienz. Da CPAP nicht nur eine CSA-CSR bei Patienten mit primärer Prädominanz einer OSA demaskieren, sondern auch effektiv in der Behandlung einer CSA-CSR sein kann [13], erhielten alle Patienten in unserer Studie zwei CPAPTherapienächte vor Einleitung der ASV-Therapie. Insofern stellen die untersuchten Patienten eine streng selektierte Gruppe dar, die gekennzeichnet ist durch eine Cheyne-Stokes-Atmung, die unter CPAP persistiert oder eindeutiger zu Tage tritt.

Die Tatsache, dass nur Patienten mit unter ASV normalisiertem AHI und einer Therapieadhärenz von mindestens $4 \mathrm{~h} / \mathrm{Nacht}$ eingeschlossen wurden, erklärt ebenfalls die kleine Studienpopulation. Aufgrund der geringen Patientenzahl in den Subgruppen ist die Aussage zur fehlenden Signifikanz der BNP-Absenkung in der CSA-CSR-Gruppe mit gewisser Vorsicht zu sehen. Die fehlende Absenkung des BNP bei einem Patienten mit guter Compliance und AHI-Normalisierung bei reiner CSA-CSR ist nicht erklärbar und beeinflusst das Gesamtergebnis der BNP-Senkung für diese Subgruppe. Unabhängig davon weisen beide Gruppen unter ASV-Therapie im Mittel einen nahezu identischen BNP-Wert auf, während der prätherapeutische Wert in der CSA-CSR + OSAGruppe signifikant höher ist, ähnlich den AHI-Werten.

Da lediglich eine CPAP-Titration bzw. -Therapie über 2 Nächte durchgeführt wurde, kann keine Aussage dazu gemacht werden, ob eine reine CPAP-Therapie schon zu einer BNP-Reduktion, insbesondere in der Subgruppe mit begleitenden Obstruktionen, geführt hätte. Allerdings war die Beantwortung dieser Fragestellung nicht die primäre Zielsetzung der Untersuchung.

Weiterhin ist die Klassifikation der Hypopnoen allein aufgrund von Flusslimitationen schwierig und mit einem Unsicherheitsfaktor behaftet, da auch bei primär zentraler Genese der Hypo- 
pnoen pharyngeale Einengungen auftreten können [43]. Die Zuordnung der Patienten zu einer reinen zentralen Atemstörung oder einer zentralen Atemstörung mit obstruktiven orientierte sich deshalb im Wesentlichen an den Apnoen und zusätzlicher Video-Auswertung.

Die Untersuchung kann nur bedingt eine Aussage zu einem möglichen Stufenkonzept in der Therapie der zentralen Schlafapnoe vom Cheyne-Stokes-Typ, insbesondere bei begleitender obstruktiver Komponente, geben. Insgesamt zeigt sich im untersuchten Gesamtkollektiv der behandlungspflichtigen Patienten mit schlafbezogener Atemstörung nur eine geringe Zahl von Patienten mit Persistenz einer Cheyne-Stokes-Atmung bei Anwendung des Konzeptes einer CPAP-Titration, insbesondere bei begleitender obstruktiver Atemstörung, vor Einleitung einer ASV-Therapie. Deshalb kann zumindest geschlussfolgert werden, dass vor Initiierung einer ASV-Therapie ein CPAP-Therapieversuch vorausgehen sollte. Dabei erweist sich der Einsatz eines Auto-adjust-Gerätes mit FOT-Signal zumindest in einer Titrationsnacht nicht als negativ, wenn auf dieser Basis eine weitere Festdrucktherapienacht durchgeführt wird. Ob die initiale CPAP-Therapie sich auf 2 Nächte beschränken oder gegebenenfalls auf einen Zeitraum von 6 Wochen bis 3 Monaten ausgedehnt werden muss, um Spätresponder zu erfassen, muss offen bleiben.

Für weitere Aussagen bezüglich der BNP-Reduktion durch ASV in unterschiedlichen Subgruppen (CSA-CSR mit und ohne OSA bei rein diastolischer Herzinsuffizienz vs. leicht vs. schwergradige Herzinsuffizienz) sind größere Patientenkollektive und längere Beobachtungszeiten erforderlich. Dabei ist zu berücksichtigen, dass sich in Abhängigkeit von einer wenn auch nur geringen EFVerbesserung eine Änderung in der dominanten Atemstörung in Form eines Shift von der Cheyne-Stokes-Atmung zu einer vorherrschend obstruktiven Atemstörung ergeben kann [44].

\section{Schlussfolgerung}

ASV kann den AHI bei Patienten, die eine Cheyne-Stokes-Atmung mit oder ohne begleitende obstruktive Ereignisse bei milder systolischer und diastolischer Herzinsuffizienz aufweisen, normalisieren. Darüber bewirkt eine ASV-Therapie eine signifikante Reduktion von erhöhten BNP-Spiegeln. Dies legt nahe, dass eine effektive Beseitigung schlafbezogener Atemstörungen in dieser Gruppe der Herzinsuffizienzpatienten die kardiale Funktion verbessert.

Eine CSA-CSR bei Herzinsuffizienzpatienten mit einer LVEF > $40 \%$ ist klinisch relevant und bedarf der weiteren Aufmerksamkeit und Untersuchung in kontrollierten Studien, ebenso wie der Einfluss begleitender obstruktiver Ereignisse auf BNP-Erhöhungen bei CSA-CSR.

\section{Abkürzungen}

VHF: Vorhofflimmern; AHI: Apnoe-Hypopnoe-Index; ASV: Adaptive Servoventilation; BMI: Body mass index; BNP: brain natriuretic peptide; CAI: Centraler Apnoe-Index; CAHI: Centraler apnoe-Hypopnoe-Index; CANPAP: Canadian Continuous Positive Airway Pressure for Patients with Central Sleep Apnea and Heart Failure Trial; CPAP: continuous positive airway pressure; CSA: Zentrale Schlafapnoe; CSA-CSR: Zentrale Schlafapnoe und Cheyne-Stokes-Atmung; EEG: Elektroenzephalogramm; ESS: Epworth Sleepiness Scale; LA: Linker Vorhof; LVEF: linksventrikuläre Ejek- tionsfraktion; MAI: Gemischter Apnoe-Index; NREM: Non-rapid eye movement; NT-pro-BNP: N-terminal pro-brain natriuretic peptide; REM: Rapid eye movement; OAI: obstruktiver ApnoeIndex; OSA: obstruktive Schlafapnoe; SAS: Schlafbezogene Atemstörung; UNE: Urin Norepinephrin Ausscheidung

\section{Interessenkonflikte \\ $\nabla$}

M. Westhoff erhält Vortragshonorare der Firmen H \& L und ResMed GmbH \& Co. KG.

M. Arzt erhält Forschungsunterstützung sowie Vortragshonorare der Firmen Respironics, Inc. (d/b/a Philips Healthcare) und ResMed GmbH \& Co. KG.

P. Litterst gibt an, dass kein Interessenkonflikt besteht.

\section{Literatur}

1 Vazir A, Hastings PC, Dayer M et al. A high prevalence of sleep disordered breathing in men with mild symptomatic chronic heart failure due to left ventricular systolic dysfunction. Eur J Heart Fail 2007; 9: $243-$ 250

2 Oldenburg O, Lamp B, Töpfer V et al. Prevalence of sleep-related breathing disorders in ischemic and non-ischemic heart failure. Dtsch Med Wochenschr 2007; 132: 661 -666

3 Ferrier K, Campbell A, Yee B et al. Sleep-disordered breathing occurs frequently in stable outpatients with congestive heart failure. Chest 2005; $128: 2116-2122$

4 Oldenburg O, Lamp B, Faber L et al. Sleep-disordered breathing in patients with symptomatic heart failure: a contemporary study of prevalence in and characteristics of 700 patients. Eur J Heart Fail 2007; 9: $251-257$

5 Tkacova R, Niroumand M, Lorenzi-Filho G et al. Overnight shift from obstructive to central apneas in patients with heart failure: role of PCO2 and circulatory delay. Circulation 2001; 103: 238-243

6 Ryan CM, Bradley TD. Periodicity of obstructive sleep apnea in patients with and without heart failure. Chest 2005; 127: 536-542

7 Kasai T, Narui K, Dohi T et al. Prognosis of patients with heart failure and obstructive sleep apnea treated with continuous positive airway pressure. Chest 2008; 133: 690-696

8 Egea CJ, Aizpuru F, Pinto JA et al. Spanish Group of Sleep Breathing Disorders: Cardiac function after CPAP therapy in patients with chronic heart failure and sleep apnea: A multicenter study. Sleep Med 2008; 9: 660-666

9 Mansfield DR, Gollogly NC, Kaye DM et al. Controlled trial of continuous positive airway pressure in obstructive sleep apnea and heart failure. Am J Respir Crit Care Med 2004; 169: 361 - 366

10 Khayat RN, Abraham WT, Patt B et al. Cardiac effects of continuous and bilevel positive airway pressure for patients with heart failure and obstructive sleep apnea: a pilot study. Chest 2008; 134: 1162 -1168

11 Kaneko Y, Floras JS, Usui $\mathrm{K}$ et al. Cardiovascular effects of continuous positive airway pressure in patients with heart failure and obstructive sleep apnea. N Engl J Med 2003; 348: 1233 - 1241

12 Bradley TD, Logan AG, Kimoff RJ et al. CANPAP Investigators. Continuous positive airway pressure for central sleep apnea and heart failure. $\mathrm{N}$ Engl J Med 2005; 353: 2025 - 2033

13 Arzt M, Floras JS, Logan AG et al. CANPAP Investigators. Suppression of central sleep apnea by continuous positive airway pressure and transplant-free survival in heart failure: a post hoc analysis of the Canadian Continuous Positive Airway Pressure for Patients with Central Sleep Apnea and Heart Failure Trial (CANPAP). Circulation 2007; 115: $3173-$ 3180

14 Sin DD, Logan AG, Fitzgerald FS et al. Effects of continuous positive airway pressure on cardiovascular outcomes in heart failure patients with and without Cheyne-Stokes respiration. Circulation 2000; 102: $61-66$

15 Teschler H, Döhring J, Wang YM et al. Adaptive pressure support servoventilation: a novel treatment for Cheyne-Stokes respiration in heart failure. Am J Respir Crit Care Med 2001; 164: 614-619

16 Pepperell JC, Maskell NA, Jones DR et al. A randomized controlled trial of adaptive ventilation for Cheyne-Stokes breathing in heart failure. Am J Respir Crit Care Med 2003; 168: 1109-1114 
17 Phillippe C, Stoica-Herman M, Drouot X et al. Compliance with and effectiveness of adaptive servoventilation versus continuous positive airway pressure in the treatment of Cheyne-Stokes respiration in heart failure over a six month period. 2006; 92: 337-342

18 Allam JS, Olson EJ, Gay PC et al. Efficacy of adaptive servoventilation in treatment of complex and central sleep apnea syndromes. Chest 2007; 132: $1839-1846$

19 Zhang XL, Yin KS, Li XL et al. Efficacy of adaptive servoventilation in patients with congestive heart failure and Cheyne-Stokes respiration. Chin Med J 2006; 119: 622-627

20 McDonagh TA, Robb SD, Murdoch DR et al. Biochemical detection of leftventricular systolic dysfunction. Lancet 1998; 351: 9-13

21 Lubien E, DeMaria A, Krishnaswamy P et al. Utility of B-natriuretic peptide in detecting diastolic dysfunction: comparison with Doppler velocity recording. Circulation 2002; 105: 595-601

22 Maisel A, Mueller C, Adams K Jr. et al. State of the art: using natriuretic peptide levels in clinical practice. Eur J Heart Fail 2008; 10: 842 - 839

23 Dickstein K, Cohen-Solal A, Fillipatos G et al. ESC guidelines for the diagnosis and treatment of acute and chronic heart failure 2008. Eur Heart J 2008; 29: $2388-2442$

24 Rechtschaffen A, Kales A. A manual of standardized terminology, techniques and scoring system for sleep stages of human subjects. Bethesda, Maryland: U.S. Department of Health, Education, and Welfare Public Health service - National Institutes of Health, 1968

25 Sleep-related breathing disorders in adults: recommendations for syndrome definition and measurement techniques in clinical research. The Report of an American Academy of Sleep Medicine Task Force. Sleep 1999; 22: 667-689

26 Floras JS. Sleep apnea in heart failure: implications of sympathetic nervous system activation for disease progression and treatment. Curr Heart Fail Rep 2005; 2: 212 - 217

27 Solin P, Kaye DM, Little PJ et al. Impact of sleep apnea on sympathetic nervous system activity in heart failure. Chest 2003; 123: 1119-1126

28 El-Solh AA, Bozkanat E, Mador J et al. Association between plasma endothelin-1 levels and Cheyne-Stokes respiration in patients with congestive heart failure. Chest 2002; 121: 1928-1934

29 Carmona-Bernal C, Quintana-Gallego E, Villa-Gil M et al. Brain natriuretic peptide in patients with congestive heart failure and central sleep apnea. Chest 2005; 127: 1667-1673

30 Christ M, Sharkova Y, Fenske $H$ et al. Brain natriuretic peptide for prediction of Cheyne-Stokes respiration in heart failure patients. Int J Cardiol 2007; 116: $62-69$
31 Steg PG, Joubin L, McCord J et al. B-type natriuretic peptide and echocardiographic determination of ejection fraction in the diagnosis of congestive heart failure in patients with acute dyspnea. Chest 2005; 128: $21-29$

32 Pfister M, Buser P, Rickli $H$ et al. for the TIME-CHF Investigators: BNPguided vs. symptom-guided heart failure therapy: the trial of intensified vs standard medial therapy in elderly patients with congestive heart failure (TIME-CHF) randomized trial. JAMA 2009; 301: 383-392

33 Oldenburg $O$, Lamp B, Bitter T et al. Adaptive servoventilation improves cardiac function in patients with chronic heart failure and Cheyne-Stokes respiration. Eur J Heart Fail 2008; 10: 581 - 586

34 Wang H, Parker JD, Newton GE et al. Influence of obstructive sleep apnea on mortality in patients with heart failure. J Am Coll Cardiol 2007; 49: $1625-1631$

35 Eskafi M, Cline C, Nilner $M$ et al. Treatment of sleep apnea in congestive heart failure with a dental device: the effect on brain natriuretic peptide and quality of life. Sleep Breath 2006; 10: 90-97

36 Chan J, Sanderson J, Chan $W$ et al. Prevalence of sleep-disordered breathing in diastolic heart failure. Chest 1997; 111: 1488 - 1493

37 Javaheri S. Sleep disorders in systolic heart failure: a prospective study of 100 male patients. The final report. Int J Cardiol 2006; 106: 21 - 28

38 Fietze I, Blau A, Glos $M$ et al. Bi-level positive pressure ventilation and adaptive servo ventilation in patients with heart failure and CheyneStokes respiration. Sleep Med 2008; 9: 652 -659

39 Oldenburg O, Lamp B, Freivogel $\mathrm{K}$ et al. Low night-to-night variability of sleep disordered breathing in patients with stable congestive heart failure. Clin Res Cardiol 2008; 97: 836 - 842

40 Tkacova R, Wang H, Bradley TD. Night-to-night alterations in sleep apnea type in patients with heart failure. J Sleep Res 2006; 15: 321 - 328

41 Vazir A, Hastings PC, Papaioannou I et al. Variation in serverity and type of sleep-disordered breathing throughout 4 nights in patients with heart failure. Respir Med 2008; 102: 831-839

42 Apelt S, Canisius S, Cassel W et al. Die diagnostische Genauigkeit der Polygraphie bei Patienten mit Herzinsuffizienz. Ein Vergleich zur Polysomnographie. Somnologie 2009; 13: 221-227

43 Sankri-Tarbichi AG, Rowley JA, Badr MS. Expiratory pharyngeal narrowing during central hypocapnic hypopnea. Am J Respir Crit Care Med 2009; 179: 313-319

44 Ryan CM, Floras JS, Logan AG et al. Shift in sleep apnoea type in heart failure patients in the CANPAP trial. Eur Respir J 2010; 35: 592 - 597 